\title{
(6) OPEN ACCESS \\ Dose-response association of screen time-based sedentary behaviour in children and adolescents and depression: a meta-analysis of observational studies
}

\author{
Mingli Liu, ${ }^{1,2}$ Lang $\mathrm{Wu}^{3,4}$ Shuqiao Yao
}

\begin{abstract}
- Additional material is published online only. To view please visit the journal online (http://dx.doi.org/10.1136/ bjsports-2015-095084).

${ }^{1}$ Medical Psychological Institute, Second Xiangya Hospital of Central South University, Changsha, Hunan, China

${ }^{2}$ School of Education, Hunan University of Science and Technology, Xiangtan, Hunan, China

${ }^{3}$ Center for Clinical and Translational Science, Mayo Clinic, Rochester, Minnesota, USA

${ }^{4}$ Division of Epidemiology, Department of Medicine, Vanderbilt Epidemiology Center, Vanderbilt University School of Medicine, Nashville, Tennessee, USA
\end{abstract}

\section{Correspondence to} Professor Shuqiao Yao, Medical Psychological Institute, Second Xiangya Hospital of Central South University, 139 Middle Renmin Road Changsha, Hunan 410011, P.R.China;

shuqiaoyao@163.com

Accepted 17 October 2015 Published Online First 9 November 2015

\section{(1) crossanak}

To cite: Liu M, Wu L, Yao S. Br J Sports Med 2016;50:1252-1258.

\begin{abstract}
Background Depression represents a growing public health burden. Understanding how screen time (ST) in juveniles may be associated with risk of depression is critical for the development of prevention and intervention strategies. Findings from studies addressing this question thus far have been inconsistent. Therefore, we conducted a comprehensive systematic review and meta-analysis of data related to this question.
\end{abstract}

Methods The meta-analysis was conducted in accordance with the PRISMA guideline. We searched the electronic databases of PubMed, Web of Science and EBSCO systematically (up to 6 May 2015). OR was adopted as the pooled measurement of association between ST and depression risk. Dose-response was estimated by a generalised least squares trend estimation.

Results Twelve cross-sectional studies and four longitudinal studies (including 1 cohort study) involving a total of 127714 participants were included. Overall, higher ST in preadolescent children and adolescents was significantly associated with a higher risk of depression $(\mathrm{OR}=1.12 ; 95 \% \mathrm{Cl} 1.03$ to 1.22). Screen type, age, population and reference category acted as significant moderators. Compared with the reference group who had no ST, there was a non-linear dose-response association of ST with a decreasing risk of depression at $\mathrm{ST}<2 \mathrm{~h} /$ day, with the lowest risk being observed for $1 \mathrm{~h} /$ day (OR=0.88; $95 \% \mathrm{Cl} 0.84$ to 0.93 ).

Conclusions Our meta-analysis suggests that ST in children and adolescents is associated with depression risk in a non-linear dose-response manner.

\section{INTRODUCTION}

With advances in technology, screen time (ST), including watching television, using a computer and playing video games, is becoming a central component of the daily lives of young children and adolescents and their most common sedentary behaviour, ${ }^{1}$ which is a construct separate from simple lack of physical activity ${ }^{2}$ and defined as any waking behaviour characterised by an energy expenditure of no more than 1.5 metabolic equivalent tasks while in a sitting or reclining posture. ${ }^{3}$ It has been estimated that infants aged 8-18 years in the USA spend an average of more than $7 \mathrm{~h} /$ day watching television, using a computer and playing video games. ${ }^{4}$ Given that this trend may continue to increase, it is important that we understand the possible impacts of extensive ST on children and adolescents.

The displacement hypothesis ${ }^{5}{ }^{6}$ suggests that time spent on screen-based activities may replace time participating in more productive and/or active activities, especially activities involving physical movement and interpersonal communication, and thus may affect the physical and mental health of young people. Concordant with this hypothesis, ST-based sedentary behaviour has been associated with risks of a variety of diseases, including cancer, ${ }^{7}$ cardiovascular disease, ${ }^{7}$ obesity, ${ }^{8}$ sleep problems, diminished psychological well-being ${ }^{9}$ and depression. ${ }^{10} 11$

Depression is defined as a cluster of specific symptoms with associated impairment, ${ }^{12}$ which represents a global public health burden, even for children and adolescents. Depression in juveniles is associated with substantial functional impairment and psychological health problems, ${ }^{12}{ }^{13}$ such as substance abuse ${ }^{14}$ impaired psychosocial functioning ${ }^{15}$ and suicide risk, ${ }^{16}$ as well as relevant to a series of psychological health disorders in later adult life. ${ }^{17}$ To evaluate whether ST-based sedentary behaviour may be associated with risk of depression in children and adolescents is critical in better guiding appropriate prevention and intervention strategies, in the hope of decreasing the burden from depression.

Depressed mood, depression syndromes and depression disorders reflect three levels of depression. ${ }^{18}$ In practice, levels of depression in juveniles have been assessed mainly by way of self-reported, parent-reported and teacher-reported depression scales, such as the Beck Depression Inventory (BDI) ${ }^{19}$ the Center for Epidemiologic Studies Depression Scale (CES-D) ${ }^{20} 21$ and the Children's Depression Inventory (CDI). ${ }^{22}$ Each has a different rubric regarding levels or symptoms of depression. For instance, the traditional cut-off of the CES-D for indication of depression is 16; however, some have used 23 to indicate probable depression. ${ }^{23}$

The findings of studies examining the potential association between ST and risk of depression in juveniles have been inconsistent. Most studies reported a linear association of ST with depression; for example, while some reported a significant positive association between computer use and depression, ${ }^{24-26}$ others have suggested negative ${ }^{27}$ or null ${ }^{28}$ associations. These contradictions may be related to the use of different methodologies, including different measures and populations. In recent years, a growing number of studies ${ }^{24-26} 2930$ have reported a non-linear association of ST in juveniles with depression. They found that groups which had excessive or no ST had a higher risk of depression level compared with the occasional or regular ST group, suggesting that appropriate ST may be associated with lower juveniles' depression 
symptoms. Similar non-linear associations were found between electronic gaming and prosocial behaviour, life satisfaction and internalising and externalising problems. ${ }^{31}$ However, since different reference groups were used in these studies, it remains unclear for the definite appropriate ST for children and adolescents for preventing depression development.

Paediatricians' ST recommendations are based largely on expert opinion or narrative reviews ${ }^{132}$ for general physical and mental health; evidence-based ST-related sedentary behaviour guidelines are lacking. A prior review of three studies ${ }^{9}$ summarised the association between screen-based sedentary behaviour and depression in adolescent girls, but did not include any quantitative assessments. A meta-analysis ${ }^{33}$ pooled estimation of association between sedentary behaviour and the risk of depression across all ages from 13 cross-sectional studies and 11 longitudinal studies, and found an increased risk of developing depression for the highest versus non-occasional/occasional sedentary behaviour $(\mathrm{RR}=1.25$; 95\% CI 1.16 to 1.35$)$. However, there was no pooled estimation of association between ST and depression for children and adolescents or any other special ages. Also, there was no dose-response analysis to explore associations of different amount of ST with depression risk. Therefore, a more comprehensive meta-analysis is needed to clarify the dose-response association between ST in juveniles and depression risk to better elucidate the putative association.

We report a comprehensive systematic review and meta-analysis summarising all available evidence related to the association between ST and depression in juveniles. We conducted a meta-analysis of all observational studies to pool the risk of depression with ST in preadolescent children and adolescents, and quantified a dose-response association. Our primary hypothesis is that excessive ST is associated with a higher risk of depression in preadolescent children and adolescents, with a non-linear dose-response relationship. On the basis of the theory of sex differences in coping mechanisms when dealing with depression ${ }^{34}$ and the evidence that depression risk may increase with age, ${ }^{13} 35$ we hypothesised that gender and age would be moderating factors of this association.

\section{METHODS}

We reported this systematic review and meta-analysis in accordance with the PRISMA guideline ${ }^{36}$ (see Research Checklist).

\section{Search strategy.}

The electronic databases of PubMed, Web of Science and EBSCO were searched systematically (up to 6 May 2015) without restricting the population, publication type or language. The following $\mathrm{MeSH}$ terms and their combinations were used in the search: sedentary behav*, screen time, sitting time, television view*, watching television, computer use, internet use, video game*, electronic game*, depress*, children, boy*, girl*, teen*, and adolescent*. The asterisk indicates that the search was inclusive of larger words that contained the word or word fragment. We also screened the bibliographies of retrieved articles to identify additional studies.

\section{Inclusion and exclusion criteria}

Studies were included if the following criteria were fulfilled: had an observational cross-sectional, case-control or longitudinal study design (including cohort design); reported correlation index of depression with ST (including television, computer/internet use, video games), such as OR, RR or correlation coefficients; participants' average ages at the time when ST was measured were in the range of 5-18 years. Studies not meeting all inclusion criteria were excluded. Articles were also excluded if: they reported mixed sedentary behaviour, among which the association between ST and depression could not be separated; measurement of screen behaviour included other types besides ST (eg, special content such as violent video games). Two authors screened all titles and abstracts and then scrutinised potential eligible studies independently. If multiple articles reported the same research, the one with the most complete information was included.

\section{Data extraction}

Data and characteristics of included studies were extracted by two investigators independently using EpiData V.3.1 and Excel software. Any differences were resolved by discussion. Extracted characteristics included the first author's name, study design, country, publication year, study year, sample size, participants' ages and genders, outcomes, duration of follow-up for prospective studies, number of cases (dose-response), type and level of ST, ST reference category, depression assessment, correlation index of depression with ST, and matched or adjusted covariates in the statistical analysis.

\section{Quality assessment}

We referenced the MOOSE ${ }^{37}$ and $\mathrm{STROBE}^{38}$ guidelines and conducted a systematic review of tools for assessing quality in observational studies in epidemiology. ${ }^{39}$ We used the following rubric for grading study quality: one point for appropriate selection of study participants; two points for proper measurements of ST and depression, respectively; one point for appropriate methods outlined to deal with any design-specific issues (recall bias, interviewer bias for cross-sectional studies and biased loss to follow-up for longitudinal studies); one point for methods of control of confounding and one point for appropriate statistical methods.

\section{Statistical analyses}

OR was used as a measurement of the association for the most included studies (10/16) that reported ORs with 95\% CIs. The most appropriate adjusted effect sizes were used if available. We converted relevant data for studies not reporting the ORs and 95\% CIs, with multiple methods. For one study ${ }^{24}$ that reported $\mathrm{RR}$ and 95\% CI, we estimated OR from RR using the formula proposed by Zhang and Kai. ${ }^{40}$ For two studies ${ }^{41} 42$ that reported ORs with SEs or p values, we estimated 95\% CIs from SEs or $\mathrm{p}$ value. ${ }^{43}$ For one cross-sectional study and three longitudinal studies ${ }^{27} 4445$ that reported correlation coefficients, we converted the coefficients to ORs with $95 \%$ CIs. ${ }^{46}$ Finally, for one study that reported mean values with SEs, we converted them to Cohen's d value with the combined group of nongamer and $<1 \mathrm{~h} /$ day as the reference category; we then converted the values to an OR with 95\% CI (see online supplementary file S1). ${ }^{46}$ Only one effect size was selected from each report to be pooled. When an individual study reported effect sizes based on multiple screen types, we combined effect sizes and used the overall datum for the pooling analysis. If applicable, the ORs were recalculated using the $0 \mathrm{~h} /$ day or occasional/ day category as the reference category. ${ }^{47}$ One study ${ }^{48}$ used a reference group with an ST $>2 \mathrm{~h} /$ day, and we recalculated the OR and $95 \% \mathrm{CI}$ as inverses. ${ }^{46}$ The method proposed by Hamling et $a l^{49}$ was used to combine multiple categories' effect sizes. Reports stratified by gender were treated as separate reports. We used a DerSimonian and Laird random-effects model to attain an overall OR and 95\% CI because most of the studies included in this meta-analysis were not functionally identical. 
Dose-response association was estimated by a generalised least squares trend estimation. ${ }^{47}{ }^{50}$ Data were extracted from eligible studies that reported multiple levels of ST with corresponding ORs and 95\% CIs, as well as distributions of cases and controls. Two studies ${ }^{25} 29$ reported only multiple levels of ST with distributions of cases and controls, and we computed unadjusted ORs and 95\% CIs of their data based on the exposure distributions of cases and controls. The crude ORs reported in this meta-analysis are within $10 \%$ of the adjusted ORs, indicating that they are approximately equal. ${ }^{47}$ We then examined both linear and non-linear associations of ST with depression risk with a two-stage random-effects dose-response analysis. ${ }^{51}$ We tested the non-linear association by hypothesising that the coefficient of the second and the third splines were both equal to zero.

Heterogeneity between studies was evaluated with the Q statistic and quantified by $\mathrm{I}^{2}$. To test the robustness of the results, we performed sensitivity analyses. We also conducted subgroup analyses to explore the sources of the heterogeneity. On the basis of the literature, the following six potential moderators were examined: screen type, gender, age, population, reference category and study quality. Qbetween values of subgroups were calculated $\left(\mathrm{Q}_{\text {total }}-\mathrm{Q}_{\text {within }}\right)$ and were tested for significance to determine group differences. ${ }^{46}$ Publication bias was detected by funnel plot asymmetry, and measured with Begg's2 and Egger's ${ }^{53}$ tests. Further adjustment for publication bias was evaluated by the trim and fill method if significant publication bias was found. ${ }^{54}$ Statistical significance was defined as $\mathrm{p}<0.05$. Statistical analyses were performed with STATA V.12 software (StataCorp LP, College Station, Texas, USA).

\section{RESULTS}

\section{Characteristics of the included studies}

Our comprehensive literature search and screen indicated that

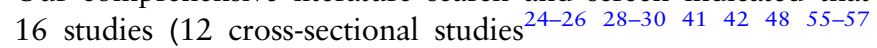
and 4 longitudinal studies, ${ }^{27} 44 \quad 45 \quad 58$ including 1 cohort study ${ }^{58}$ ), from a total of 21 reports, including a total of 127714 participants (115 241 in cross-sectional studies and 12473 in longitudinal studies), met our inclusion criteria (figure 1).

The characteristics of the included studies are summarised in online supplementary table S1 (see online supplementary table S1). The sample sizes varied widely across cross-sectional studies (from 160 to 75066 ) and longitudinal studies (from 198 to 10347$)$. All of the studies involved both male and female participants; gender groups were analysed separately in five cross-sectional studies. The mean participant age was $<14$ years in six studies and $>14$ years in six studies; mean age was not reported in the four remaining studies. Four studies were conducted in Europe, five in North America, two in Australia and five in Asia.

Several reference categories were used in the 16 analysed studies. Four studies used $0 \mathrm{~h} /$ day or occasional as the reference category, three used $1 \mathrm{~h} /$ day (cumulative), four used $2 \mathrm{~h}$ /day (cumulative) and others analysed continuous ST. Three studies did not adjust for any covariates. Controlled factors included age $(n=6)$, gender $(n=7)$, grade $(n=4)$, body mass index $(n=2)$, socioeconomic status $(n=5)$, parents' education $(n=4)$, race $(n=2)$ and smoking $(n=2)$. The average quality score of all included studies was 4.44 , with nine studies obtaining a quality score $\geq 5$ (see online supplementary table S2).

\section{ST and depression risk}

As presented in figure 2, the overall pooled OR was 1.12 (95\% CI 1.03 to $1.22 ; \mathrm{p}=0.007)$ with high heterogeneity

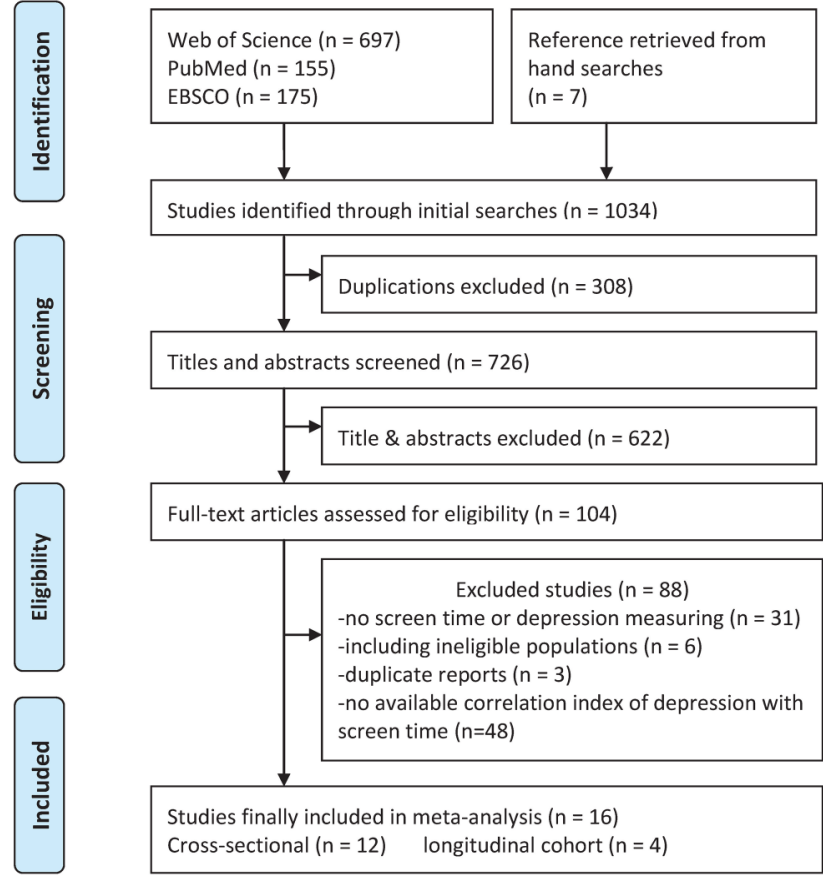

Figure 1 Flow chart of article screening process.

$\left(\mathrm{I}^{2}=82.5 \%\right)$. The combined OR was 1.19 (95\% CI 1.10 to $1.30 ; \mathrm{p}<0.001)$ for cross-sectional studies and $0.88(95 \% \mathrm{CI}$ 0.67 to $1.14 ; \mathrm{p}=0.327$ ) for longitudinal studies. Both study types had high heterogeneity $\left(\mathrm{I}^{2}\right.$ of $73.8 \%$ and $90.4 \%$, respectively).

\section{Subgroup and sensitivity analyses}

Subgroup analyses are shown in table 1. The association was moderated by age, population and reference group.

In sensitivity analyses in which studies were omitted one by one, no one study caused heterogeneity across the studies or influenced the results markedly (see online supplementary figure S1). There were no significant changes for results of overall studies and cross-sectional specific studies when the converted studies $^{27} 44 \quad 455557$ were excluded from analyses. For longitudinal studies, only one study with a significant effect size in the reverse direction remained (table 2).

To identify the potential effects of adjusted variables on the association, we carried out several additional sensitivity analyses by excluding studies that did not adjust for any covariates, studies without any adjustment for family or socioeconomic status and studies without any adjustment for health risk behaviour, respectively. Only one sensitivity analysis of excluding studies that did not have any adjustment for family or socioeconomic status led to a significant change of effect size (OR from 0.88 to 1.07 ) in longitudinal studies (see table 2).

\section{Publication bias}

Begg's rank correlation test $(p=0.487)$ and Egger's linear regression test $(p=0.642)$ suggested that there was no significant publication bias (see online supplementary figure S2).

Dose-response association between ST and depression risk Four studies $^{25} 262930$ (7 reports) were used for the doseresponse analysis. The analysis showed a curvilinear relationship between ST and risk of depression $(p<0.001$ for non-linearity; figure 3). There was a dose-response association of ST with a 
Figure 2 Forest plot of the association between depression risk and screen time (hours/day) in children and adolescents by study design. OR of depression risk for higher daily screen time compared with reference groups and corresponding $95 \% \mathrm{Cl}$ ( $F$, female; $M$, male).

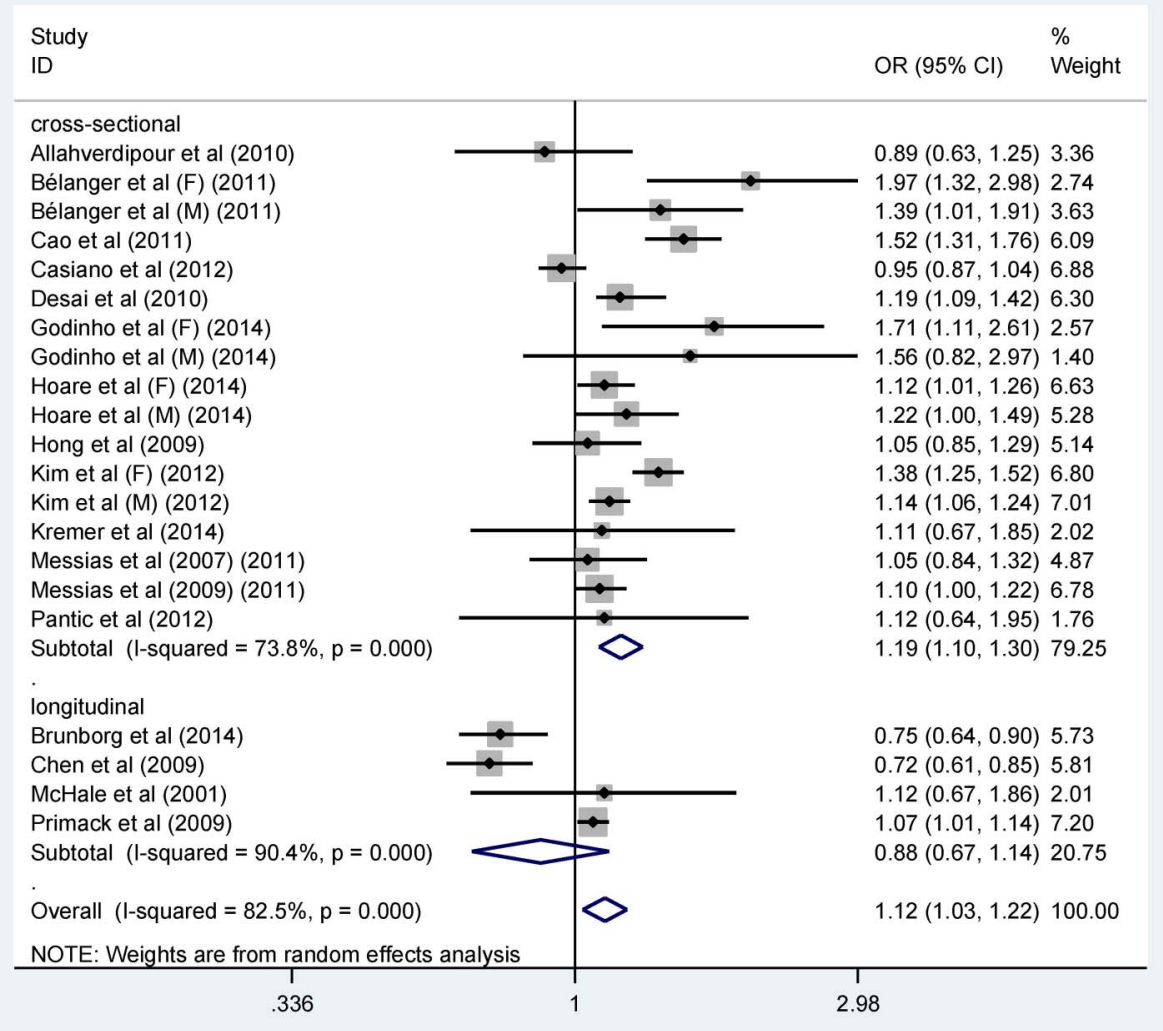

decreasing risk of depression when STs were $<1 \mathrm{~h} /$ day further when ST were. Inversely, there was a continuously increasing risk of depression with increasing ST beyond $1 \mathrm{~h}$ /day. Compared with the reference group who had no ST, the estimated ORs of depression risk were 0.92 (95\% CI 0.89 to 0.95 ) for $0.5 \mathrm{~h} /$ day

Table 1 Moderation analyses for screen time-depression association

\begin{tabular}{|c|c|c|c|c|}
\hline Factor & $\mathrm{N}$ & OR $(95 \% \mathrm{Cl})$ & $\mathrm{p}$ Value & $\mathrm{I}^{2}(\%)$ \\
\hline \multicolumn{5}{|l|}{ Gender: $Q_{(1)}=0$} \\
\hline Male & 5 & 1.16 (1.08 to 1.24$)$ & $<0.001$ & 0.0 \\
\hline Female & 5 & 1.25 (0.94 to 1.66$)$ & 0.132 & 93.2 \\
\hline \multicolumn{5}{|c|}{ Age (years): $Q_{(1)}=9.89^{* *}$} \\
\hline$<14$ & 8 & 1.25 (1.09 to 1.43$)$ & 0.001 & 56.1 \\
\hline$>14$ & 8 & $1.10(0.97$ to 1.26$)$ & 0.151 & 80.5 \\
\hline \multicolumn{5}{|c|}{ Study quality score: $\mathrm{Q}_{(1)}=0.85$} \\
\hline$\geq 5$ & 12 & 1.14 (1.05 to 1.24$)$ & 0.003 & 69.1 \\
\hline$<5$ & 9 & $1.10(0.92$ to 1.31$)$ & 0.313 & 89.7 \\
\hline \multicolumn{5}{|c|}{ Population: $\mathrm{Q}_{(3)}=11.5^{* *}$} \\
\hline European & 6 & 1.33 (0.90 to 1.96$)$ & 0.155 & 85.1 \\
\hline North American & 6 & $1.06(0.996$ to 1.14$)$ & 0.064 & 45.4 \\
\hline Australian & 3 & 1.14 (1.04 to 1.26$)$ & 0.006 & 0.0 \\
\hline Asian & 6 & $1.10(0.90$ to 1.34$)$ & 0.345 & 91.6 \\
\hline \multicolumn{5}{|c|}{ Reference category (hours/day): $Q_{(3)}=18.64^{* * *}$} \\
\hline 0 & 5 & $1.06(0.99$ to 1.14$)$ & 0.086 & 56.1 \\
\hline$\leq 1$ & 4 & 1.15 (0.99 to 1.34$)$ & 0.066 & 78.2 \\
\hline$\leq 2$ & 6 & 1.33 (1.13 to 1.57$)$ & 0.001 & 68.5 \\
\hline Continuous & 4 & $0.79(0.67$ to 0.92$)$ & 0.003 & 33.4 \\
\hline
\end{tabular}

of ST, 0.88 (95\% CI 0.84 to 0.93 ) for $1 \mathrm{~h} /$ day, 0.91 (95\% CI 0.87 to 0.96 ) for $1.5 \mathrm{~h} /$ day, 0.99 (95\% CI 0.94 to 1.04 ) for $2 \mathrm{~h} /$ day, 1.08 (95\% CI 1.03 to 1.14 ) for $2.5 \mathrm{~h} /$ day, 1.19 (95\% CI 1.13 to 1.26 ) for $3 \mathrm{~h} /$ day, 1.46 (95\% CI 1.36 to 1.57$)$ for $4 \mathrm{~h} /$ day, and 1.80 (95\% CI 1.60 to 2.02 ) for $\geq 5 \mathrm{~h} /$ day.

\section{Time of television, video games, or computer use and depression risk}

Thirteen studies (20 reports) demonstrated an association between time spent on watching television, playing video games or using a computer and depression risk (figure 4). The pooled OR estimates were 1.01 (95\% CI 0.87 to 1.16) for watching television, 0.89 (95\% CI 0.74 to 1.06 ) for playing video games and 1.28 (95\% CI 1.10 to 1.47 ) for computer use. Significance tests of effect size showed that only computer use was related to depression risk $(p=0.001)$. Heterogeneity of the association between computer time and depression risk was caused mostly by two large studies. When we omitted them, ${ }^{26}{ }^{28}$ there was no heterogeneity for the pooled estimate effect $\left(\mathrm{I}^{2}\right.$ from $82.8 \%$ to $0 \%)$. Exclusion of the two studies led to a higher effect size $(\mathrm{OR}=1.41 ; 95 \%$ CI 1.29 to 1.54$)$. Heterogeneity of the association between television time and depression risk was caused mostly by one study. When we omitted this study, ${ }^{27}$ there was much less heterogeneity for the pooled estimate effect $\left(\mathrm{I}^{2}\right.$ from $86.1 \%$ to $35.5 \%)$. Exclusion of this study led to an inverse effect size $(\mathrm{OR}=1.04$; 95\% CI 0.96 to 1.13$)$. No study significantly caused heterogeneity in the video games group.

\section{DISCUSSION}

In the present meta-analysis, we quantitatively evaluated the association of ST with depression risk in preadolescent children and adolescents. Compared with the reference groups, those 
Table 2 Sensitivity analysis of screen time and risk of depression in juveniles

\begin{tabular}{|c|c|c|c|c|}
\hline Variable & Number of reports & OR $(95 \% \mathrm{Cl})$ & P Value & $I^{2}, p$ Value* \\
\hline No study being excluded & 21 & 1.12 (1.03 to 1.22$)$ & 0.007 & $82.5,<0.001$ \\
\hline CS studies & 17 & 1.20 (1.10 to 1.30$)$ & $<0.001$ & $73.8,<0.001$ \\
\hline LS studies & 4 & $0.88(0.67$ to 1.14$)$ & 0.327 & $90.4,<0.001$ \\
\hline Exclude the converted studies & 16 & $1.19(1.11$ to 1.29$)$ & $<0.001$ & $76.5,<0.001$ \\
\hline CS studies & 15 & 1.21 (1.11 to 1.32$)$ & $<0.001$ & $76.2,<0.001$ \\
\hline LS studies & 1 & 1.07 (1.01 to 1.14$)$ & 0.023 & - \\
\hline Exclude studies with no covariate adjustment & 18 & 1.16 (1.10 to 1.26$)$ & $<0.001$ & $80.3,<0.001$ \\
\hline CS studies & 15 & 1.21 (1.11 to 1.32$)$ & $<0.001$ & $76.2,<0.001$ \\
\hline LS studies & 3 & $0.94(0.71$ to 1.26$)$ & 0.695 & $86.8,0.001$ \\
\hline Exclude studies with no adjustment for family or socioeconomic status $†$ & 16 & 1.19 (1.10 to 1.29$)$ & $<0.001$ & $76.3,<0.001$ \\
\hline CS studies & 14 & $1.22(1.11$ to 1.34$)$ & $<0.001$ & $77.8,<0.001$ \\
\hline LS studies & 2 & 1.07 (1.01 to 1.14$)$ & 0.021 & $0.0,0.870$ \\
\hline Exclude studies with no adjustment for health risk behaviourf & 8 & 1.25 (1.12 to 1.40$)$ & $<0.001$ & $77.7,<0.001$ \\
\hline CS studies & 8 & $1.25(1.12$ to 1.40$)$ & $<0.001$ & 77.7, $<0.001$ \\
\hline LS studies & 0 & - & - & - \\
\hline
\end{tabular}

${ }^{*} \mathrm{p}$ Value for heterogeneity.

tFamily and socioeconomic status including: race/ethnicity, family structure, parental education, presence of parents, whether the family lives together, parents' job status, socioeconomic status/household income.

¥Health risk behaviour including: physical activity, body mass index, smoking, drinking, drug abuse, fruit and vegetable intake, sleep time, other sedentary behaviours.

CS, cross-sectional; LS, longitudinal study.

with more daily ST had a $12.3 \%$ increase in risk of depression. ST of approximately $1 \mathrm{~h} /$ day was associated with a reduced risk of depression, whereas beyond $2 \mathrm{~h}$ /day of ST, higher ST values were associated with a continuously increasing risk of depression. Compared with the reference group, those with $0.5,1$ and $1.5 \mathrm{~h} /$ day of ST had a $8 \%, 12 \%$ and $9 \%$ reduction in risk of depression, respectively, whereas those with $2 \mathrm{~h}$ /day of ST had an approximately equal risk of depression as those with no ST. Meanwhile, individuals with $2.5,3,4$ or $\geq 5$ h of ST per day had an $8 \%, 19 \%, 46 \%$ and $80 \%$ increased risk relative to no ST, respectively. These findings are consistent with the recommendations and guidelines of developed countries regarding limiting ST. Findings regarding the dose-response association provide a better clarification of the association between ST and

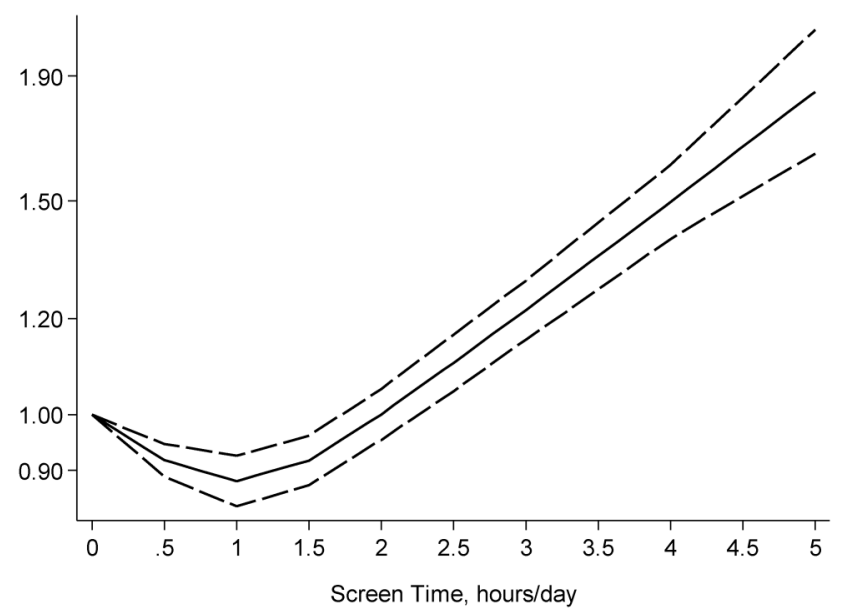

Figure 3 The dose-response relationship for the association between screen time in children and adolescents and risk of depression (solid line) as estimated by a generalised least squares trend estimation. Screen time was modelled with a restricted cubic spline in a two-stage random-effects dose-response model. The ORs are plotted on the log scale. Dashed lines represent the $95 \% \mathrm{Cls}$ for the spline model. No screen time served as the referent category. depression risk, indicating that ST in moderation may actually be associated with lower depression.

It is worth noting that an insignificant reverse association with risk of depression was detected in longitudinal studies. However, when we excluded studies with no adjustment for family or socioeconomic status, two studies with high-quality scores suggested a significantly increased risk of depression $(\mathrm{OR}=1.07 ; 95 \% \mathrm{CI} 1.01$ to 1.14$)$, with no heterogeneity. This relationship does not necessarily mean causality. There is also another possibility that previous depression state in juveniles lead to a different choose to ST. Only two longitudinal studies $^{44} 45$ reported this association with reversed results (r, $-0.07 ; \mathrm{r}, 0.03$ ). The currently available evidence is not sufficient yet to fully address the issue of reverse causality. Additional prospective studies are warranted.

Few studies have evaluated the association between ST and mental health. ${ }^{7} 9$ In a review of four studies, Temmel and Rhodes $^{59}$ suggested that psychological factors such as depression were positively associated with ST. In another review of three studies involving adolescent girls, Costigan et $a l^{9}$ found a positive association between ST and depression, as well as a meta-analysis conducted by Zhai et al. ${ }^{33}$ The pooled risk of the included cross-sectional studies in our meta-analysis was largely consistent with these previous reviews, and the findings support the notion that excessive ST is associated with a higher risk of depression in preadolescent children and adolescents.

One interesting finding in this meta-analysis is that we detected a significant curvilinear dose-response association between ST and risk of depression. Typically, guidelines and recommendations ${ }^{32} 60$ emphasise an overall positive association between ST and morbidity risk. However, the present findings suggest that when ST is limited to $0-2 \mathrm{~h} /$ day, ST is associated with a lower risk of depression, and the lowest risk is detected at ST of $1 \mathrm{~h} /$ day. Such potential benefits ${ }^{32}$ could be related to screen behaviours enhancing children's ability to read and visualise images and, consequently, improving academic performance. Alternatively, kids may benefit psychologically from processing humorous content in television, the internet and 
Figure 4 Forest plot of the association between depression risk and screen time (hours/day) in children and adolescents by screen type. OR of depression risk for higher daily screen time compared with reference groups and corresponding $95 \% \mathrm{Cl}$ (F, female; $\mathrm{CU}$, computer/internet use; $\mathrm{M}$, male; TV, television; VG/CG, video games/ computer games).

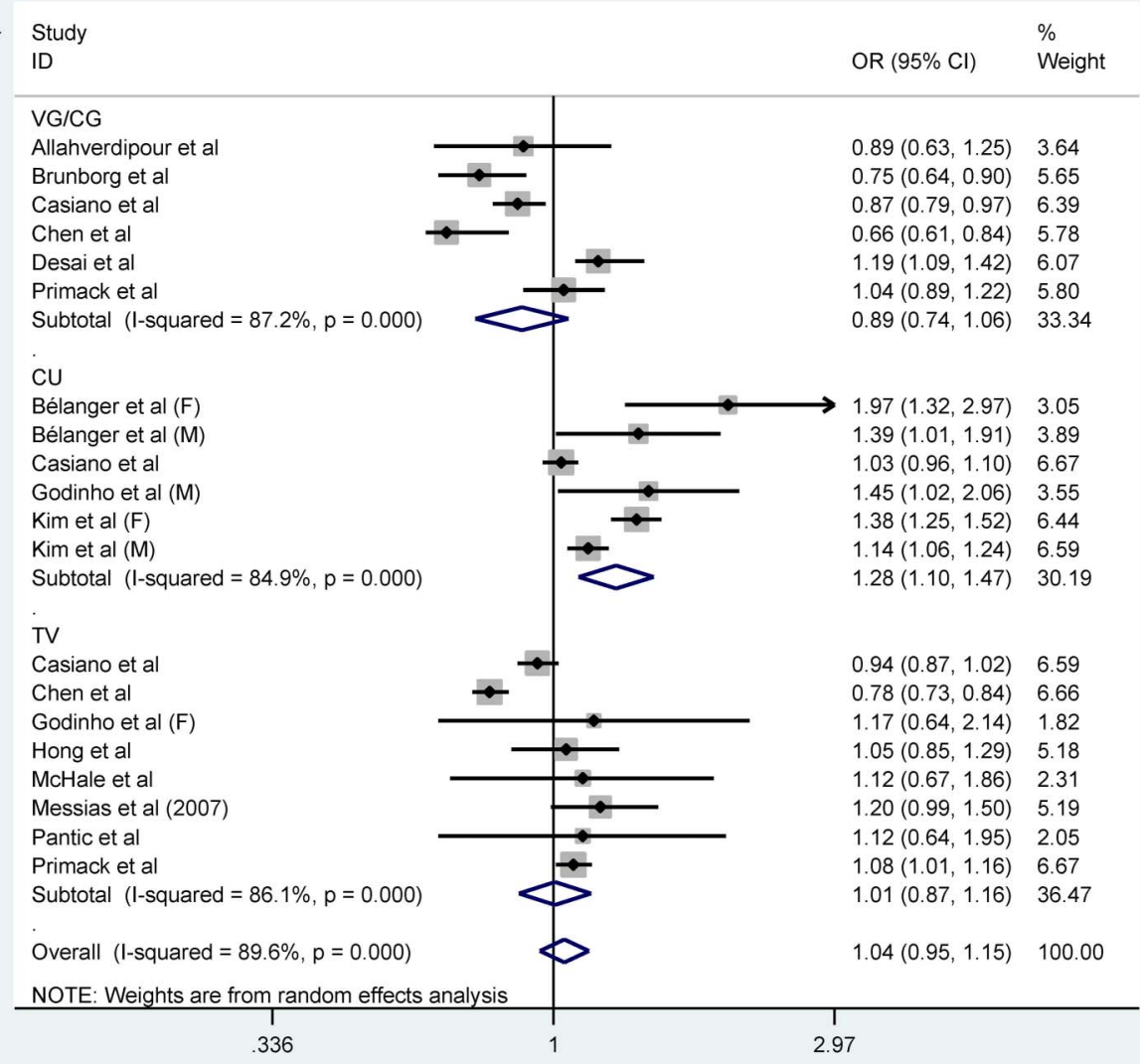

video games. ${ }^{58}$ However, it is worth noting that the small size of the reference groups may be non-comparable in other ways and have caused a misleading non-linear dose-response curve. Our studies in dose-response analysis, however, ranged from 69 to 8102 in the reference groups. Still, there may be some other uncontrolled covariates which could influence the results. Follow-up dose-response studies with complete controlling for all relevant covariates are needed to clarify this question.

Relative to the other two screen types, those who reported spending more time on the computer had a $27.6 \%$ increase in risk of depression compared with the reference group. Potentially, this effect could be due to the relative openness of the computer content, especially the internet, compared to television and video games, if youth are being exposed more readily to negative information on their computers than they would otherwise encounter. Still, there may be a possibly significant difference in other covariates between the reference groups and the exposed groups influencing the results, for example, the size of the reference groups may be small and non-comparable in other ways. Not reporting the size of multiple levels of ST in some studies precluded us from clarifying this question.

In our meta-analysis, only the pooled estimate for boys was significant. However, there was no evidence of significant heterogeneity between the two groups. This result is not consistent with a previous study ${ }^{58}$ in which a significantly lower risk of developing depression was associated with greater total media exposure (including television, videocassettes, video games and radio) in young women. It is possible that computer use, which was not assessed in their study, may underlie the different outcomes of the two studies. Also, sample size as well as other covariates may explain this result. Further studies with appropriate adjustments are needed to clarify this issue.

We observed a significant ST-depression risk in teenagers $<14$ years, but not in those over 14 years. Compared with the reference group, teenagers $<14$ years with more ST per day had a $25 \%$ increased risk of depression. The result is consistent with a previous research ${ }^{48}$ which found a significant interaction effect of age group and ST on depression (OR $=0.77 ; 95 \% \mathrm{CI}, 0.59$ to 0.99; reference, younger (10-11 years)), suggesting that higher ST was associated with higher depression risk and this was especially true for younger children. For having an inherent biological need, the younger children are more active. ${ }^{61}$ ST, however, may replace time participating in active activities, ${ }^{6}$ and thus may affect younger children more substantially. Besides, younger children have greater vulnerability to negative information from screen use, which also makes fewer opportunities for self-development and interpersonal communication, ${ }^{48}$ and thus may be more susceptible to depression.

Study population played a significant moderating role in the association between ST and depression risk. Only studies conducted in Australia demonstrated a significant correlation: those who spent more time on screen had a $14.2 \%$ increased risk of depression compared with the reference groups. No heterogeneity was found, which suggests that the result was robust in this group. After excluding one longitudinal Norwegian study, ${ }^{44}$ which reported correlation coefficients, there was a strong association between ST and depression risk for the European studies $(\mathrm{OR}=1.54 ; 95 \%$ CI 1.27 to 1.87$)$ with no evidence of significant heterogeneity $\left(\mathrm{I}^{2}=0 \%\right)$, suggesting that individuals in exposed groups have a $54.3 \%$ increase in risk of depression compared with reference individuals. No significant correlation 
was detected in North America and Asia. More studies that consider population effects are needed to clarify this issue.

After dividing the reference groups into four types, we found that relative to the $<2 \mathrm{~h} /$ day reference group, exposed groups had a remarkable $33.4 \%$ (95\% CI 1.13 to 1.57 ) increase in risk of depression. Conversely, a $21.3 \%(95 \%$ CI 0.67 to 0.92$)$ reduction in risk of depression was found in studies reporting correlation coefficients, which indicates that conversion from a correlation coefficient to an OR may somehow transform the real association. Results from data converted from correlation coefficients should be interpreted with caution.

There was no association between ST and depression risk in studies using $0 \mathrm{~h}$ or $<1 \mathrm{~h} /$ day as the reference category. This result is consistent with the curvilinear association of ST with depression risk in our dose-response analysis, indicating that the ST-depression risk association breaks at the $2 \mathrm{~h}$ /day cut-off. However, the non-significance may be caused by the smaller size of the reference groups, which made it non-comparable in other ways. Also, some other uncontrolled variables may influence the results. Further studies with proper controlling for relevant covariates are needed to clarify this issue.

In our meta-analysis, we quantified systematically the doesresponse association between ST and depression risk in preadolescent children and adolescents for the first time. The combined sample size was large. Our study supported that screen type, age, study population and reference group were potential sources of heterogeneity. The dose-response analysis showed a curvilinear association of ST with depression risk, which helped clarify the pattern of association.

Youth today are exposed to screen experience on a level never observed before. Greater efforts to disseminate information about the negative effects of too much ST in relation to risk of depression are needed to prevent negative public health outcomes of excessive ST. Such information can be distributed to parents directly or by way of educators and paediatricians. For countries or regions where screen use is not yet highly prevalent (eg, some developing countries), findings from this meta-analysis may provide a theoretical basis for development of policies or recommendations regarding appropriate ST for youth.

This study had several potential limitations. Owing to the nature of observational studies, it is difficult to exclude potential biases induced by other lifestyle factors. ${ }^{63}$ Although the data were adjusted for potential confounding variables in most of the included studies, such adjustments were variable across studies. Notably, most of the studies did not adjust for physical activity, even though physical activity has been shown to be a protective factor for mental health including depression. ${ }^{64}{ }^{65}$ Although we performed several sensitivity analyses of excluding studies without adjustment for some important covariates, there may be other potential covariates that influenced the results, such as family history of mental illness including depression. Additionally, the measures of depression were not uniform across the studies, and not all of the included studies reported estimated effects of multiple differentiated ST, limiting a comprehensive analysis. Furthermore, some other new screen types were not included in our study, such as tablets and smartphones. Further studies are needed to answer this research question. Finally, the findings in this study should be interpreted with caution; however, since $1 \mathrm{~h} /$ day of ST may be associated with a lower risk of depression, ST may be still associated with other risks. Dose-response association analyses between ST and other physical and mental health outcomes, such as obesity, heart disease and cancer, are needed to clarify this issue.

\section{CONCLUSION AND PRACTICAL IMPLICATIONS}

For preadolescents and adolescents, ST is associated with depression risk in a non-linear dose-response manner. ST is associated with a higher risk of depression when it exceeds $2 \mathrm{~h} /$ day, whereas less ST may be associated with a lower risk of depression, with the lowest risk being correlated with $1 \mathrm{~h}$ /day. Our study supports current recommendations of limiting ST to promote the health of children and adolescents.

\section{What are the findings?}

- This is the first meta-analysis estimating the dose-response association of screen time and depression in children and adolescents.

- The current meta-analyses suggested that screen time in children and adolescents was associated with depression in a non-linear dose-response manner.

- Screen time is associated with a higher risk of depression when it exceeds $2 \mathrm{~h} /$ day, whereas lesser screen time may be associated with a lower risk of depression, with the lowest risk being $1 \mathrm{~h} /$ day.

\section{How might it impact on clinical practice in the future?}

- Appropriate screen time in juveniles may be potentially beneficial for the primary prevention of depression. Further prospective studies are needed to confirm this finding.

- Further research should assess the potential influence of relevant covariates on the association between screen time in juveniles and depression risk, such as physical activity and family history of mental illness.

- Further prospective studies are needed to explore whether there exists mutual causality between screen time and depression.

Contributors $\mathrm{ML}$ and SY contributed to the conception and design. ML and LW were involved in the data analysis and all the authors interpreted the results. ML drafted the manuscript, which was revised by LW and SY. All the authors approved the version to be published.

Funding This research received no specific grant from any funding agency in the public, commercial or not-for-profit sectors.

Competing interests None declared.

Provenance and peer review Not commissioned; externally peer reviewed.

Open Access This is an Open Access article distributed in accordance with the Creative Commons Attribution Non Commercial (CC BY-NC 4.0) license, which permits others to distribute, remix, adapt, build upon this work non-commercially, and license their derivative works on different terms, provided the original work is properly cited and the use is non-commercial. See: http://creativecommons.org/ licenses/by-nc/4.0/

\section{REFERENCES}

1 Tremblay MS, LeBlanc AG, Kho ME, et al. Systematic review of sedentary behaviour and health indicators in school-aged children and youth. Int J Behav Nutr Phys Act 2011;8:98.

2 Taveras EM, Field AE, Berkey CS, et al. Longitudinal relationship between television viewing and leisure-time physical activity during adolescence. Pediatrics 2007; 119:314-19.

3 Sedentary Behaviour Research Network. Letter to the editor: standardized use of the terms "sedentary" and "sedentary behaviours". Appl Physiol Nutr Me 2012;37:540-2 
4 Rideout VJ, Foehr UG, Roberts DF. Generation M [superscript 2]: Media in the Lives of 8-to 18-Year-Olds. Henry J Kaiser Family Foundation. 2010. http://kff.org/other/ event/generation-m2-media-in-the-lives-of/ (accessed 20 Jan 2010).

5 Ohannessian CM. Media use and adolescent psychological adjustment: an examination of gender differences. J Child Fam Stud 2009;18:582-93.

6 Kraut $\mathrm{R}$, Patterson $\mathrm{M}$, Lundmark $\mathrm{V}$, et al. Internet paradox. A social technology that reduces social involvement and psychological well-being? Am Psychol 1998;53:1017-31.

7 de Rezende LF, Rodrigues Lopes M, Rey-López JP, et al. Sedentary behavior and health outcomes: an overview of systematic reviews. PLOS ONE 2014;9:e105620.

8 Benson LP, Williams RJ, Novick MB. Pediatric obesity and depression: a cross-sectional analysis of absolute BMI as it relates to children's depression index scores in obese 7- to 17-year-old children. Clin Pediatr (Phila) 2013;52:24-9.

9 Costigan SA, Barnett L, Plotnikoff RC, et al. The health indicators associated with screen-based sedentary behavior among adolescent girls: a systematic review. J Adolesc Health 2013;52:382-92.

10 Feng Q, Zhang QL, Du Y, et al. Associations of physical activity, screen time with depression, anxiety and sleep quality among Chinese college freshmen. PLOS ONE 2014;9:e100914.

11 Hamer M, Stamatakis E. Prospective study of sedentary behavior, risk of depression, and cognitive impairment. Med Sci Sport Exer 2014;46:718-23.

12 Thapar A, Collishaw S, Pine DS, et al. Depression in adolescence. Lancet 2012;379:1056-67.

13 Calles JL Jr. Depression in children and adolescents. Prim Care 2007;34:243-58; abstract vi.

14 Hersh J, Curry JF, Kaminer Y. What is the impact of comorbid depression on adolescent substance abuse treatment? Subst Abus 2014;35:364-75.

15 Gotlib IH, Lewinsohn PM, Seeley JR. Symptoms versus a diagnosis of depression: differences in psychosocial functioning. J Consult Clin Psychol 1995;63:90-100.

16 Hawton K, van Heeringen K. Suicide. Lancet 2009;373:1372-81.

17 Fergusson DM, Horwood LJ, Ridder EM, et al. Subthreshold depression in adolescence and mental health outcomes in adulthood. Arch Gen Psychiatry 2005;62:66-72.

18 Compas BE, Ey S, Grant KE. Taxonomy, assessment, and diagnosis of depression during adolescence. Psychol Bull 1993;114:323-44.

19 Beck AT, Ward CH, Mendelson $M$, et al. An inventory for measuring depression. Arch Gen Psychiatry 1961;4:561-71.

20 Radloff LS. The CES-D scale a self-report depression scale for research in the general population. App/ Psychol Meas 1977:1:385-401.

21 Wang $M$, Armour C, Wu Y, et al. Factor structure of the CES-D and measurement invariance across gender in mainland Chinese adolescents. J Clin Psychol 2013:69:966-79.

22 Wu W, Lu Y, Tan F, et al. Assessing measurement invariance of the Children's depression inventory in Chinese and Italian primary school student samples. Assessment 2012;19:506-16.

23 Radloff LS. The use of the Center for Epidemiologic Studies Depression Scale in adolescents and young adults. J Youth Adolesc 1991;20:149-66.

24 Bélanger RE, Akre C, Berchtold A, et al. A U-shaped association between intensity of Internet use and adolescent health. Pediatrics 2011;127:e330-5.

25 Godinho J, Araújo J, Barros H, et al. Characteristics associated with media use in early adolescence. Cad Saude Publica 2014;30:587-98.

26 Kim JY. The nonlinear association between Internet using time for non-educational purposes and adolescent health. J Prev Med Public Health 2012:45:37-46.

27 Chen SY, Lu L. After-school time use in Taiwan: effects on educational achievement and well-being. Adolescence 2009:44:891-909.

28 Casiano H, Kinley DJ, Katz LY, et al. Media use and health outcomes in adolescents: findings from a nationally representative survey. J Can Acad Child Adolesc Psychiatry 2012;21:296-301.

29 Messias E, Castro J, Saini A, et al. Sadness, suicide, and their association with video game and internet overuse among teens: results from the youth risk behavior survey 2007 and 2009. Suicide Life Threat Behav 2011;41:307-15.

30 Hong $X$, Li J, Xu F, et al. Physical activity inversely associated with the presence of depression among urban adolescents in regional China. BMC Public Health 2009:9:148.

31 Przybylski AK. Electronic gaming and psychosocial adjustment. Pediatrics 2014;134 e716-22.

32 American Academy of Pediatrics. Committee on Public Education. American Academy of Pediatrics: Children, adolescents, and television. Pediatrics 2001;107:423-6.

33 Zhai L, Zhang $Y$, Zhang D. Sedentary behaviour and the risk of depression: a meta-analysis. Br J Sports Med 2015;49:705-9.

34 Nolen-Hoeksema S. Sex differences in unipolar depression: evidence and theory. Psychol Bull 1987;101:259-82.

35 Jane Costello E, Erkanli A, Angold A. Is there an epidemic of child or adolescent depression? J Child Psychol Psychiatry 2006;47:1263-71.
36 Moher D, Liberati A, Tetzlaff J, et al. Preferred reporting items for systematic reviews and meta-analyses: the PRISMA statement. PLoS Med 2009;6:e1000097.

37 Stroup DF, Berlin JA, Morton SC, et al. Meta-analysis of observational studies in epidemiology: a proposal for reporting. Meta-analysis Of Observational Studies in Epidemiology (MOOSE) group. JAMA 2000;283:2008-12.

38 Von Elm E, Altman DG, Egger M, et al., STROBE Initiative. The Strengthening the Reporting of Observational Studies in Epidemiology (STROBE) statement: guidelines for reporting observational studies. Prev Med 2007:45:247-51.

39 Sanderson S, Tatt ID, Higgins JP. Tools for assessing quality and susceptibility to bias in observational studies in epidemiology: a systematic review and annotated bibliography. Int J Epidemiol 2007;36:666-76.

40 Zhang J, Kai FY. What's the relative risk? A method of correcting the odds ratio in cohort studies of common outcomes. JAMA 1998:280:1690-1.

41 Desai RA, Krishnan-Sarin S, Cavallo D, et al. Video-gaming among high school students: health correlates, gender differences, and problematic gaming. Pediatrics 2010;126:E1414-24.

42 Hoare E, Millar L, Fuller-Tyszkiewicz M, et al. Associations between obesogenic risk and depressive symptomatology in Australian adolescents: a cross-sectional study. J Epidemiol Community Health 2014;68:767-72.

43 Altman DG, Bland JM. How to obtain the confidence interval from a $P$ value. BMJ 2011;343:d2090

44 Brunborg GS, Mentzoni RA, Frøyland LR. Is video gaming, or video game addiction, associated with depression, academic achievement, heavy episodic drinking, or conduct problems? J Behav Addict 2014;3:27-32.

45 McHale SM, Crouter AC, Tucker CJ. Free-time activities in middle childhood: links with adjustment in early adolescence. Child Dev 2001;72:1764-78.

46 Borenstein M, Hedges LV, Higgins JP, et al. Introduction to meta-analysis. John Wiley \& Sons, 2009:33-186.

47 Greenland S, Longnecker MP. Methods for trend estimation from summarized dose-response data, with applications to meta-analysis. Am J Epidemiol 1992;135:1301-9.

48 Kremer $P$, Elshaug $C$, Leslie $E$, et al. Physical activity, leisure-time screen use and depression among children and young adolescents. J Sci Med Sport 2014;17:183-7.

49 Hamling J, Lee $P$, Weitkunat $R$, et al. Facilitating meta-analyses by deriving relative effect and precision estimates for alternative comparisons from a set of estimates presented by exposure level or disease category. Stat Med 2008;27:954-70.

50 Orsini N, Bellocco R, Greenland S. Generalized least squares for trend estimation of summarized dose-response data. Stata J 2006:6:40-57.

51 Orsini N, Li R, Wolk A, et al. Meta-analysis for linear and nonlinear dose-response relations: examples, an evaluation of approximations, and software. Am J Epidemiol 2012:175:66-73.

52 Begg CB, Mazumdar M. Operating characteristics of a rank correlation test for publication bias. Biometrics 1994;50:1088-101.

53 Egger M, Davey Smith G, Schneider M, et al. Bias in meta-analysis detected by a simple, graphical test. BMJ 1997;315:629-34.

54 Duval S, Tweedie R. Trim and fill: a simple funnel-plot-based method of testing and adjusting for publication bias in meta-analysis. Biometrics 2000;56:455-63.

55 Allahverdipour H, Bazargan M, Farhadinasab A, et al. Correlates of video games playing among adolescents in an Islamic country. BMC Public Health 2010;10:286.

56 Cao H, Qian Q, Weng T, et al. Screen time, physical activity and mental health among urban adolescents in China. Prev Med 2011;53:316-20.

57 Pantic I, Damjanovic A, Todorovic J, et al. Association between online social networking and depression in high school students: behavioral physiology viewpoint. Psychiatr Danub 2012:24:90-3.

58 Primack BA, Swanier B, Georgiopoulos AM, et al. Association between media use in adolescence and depression in young adulthood: a longitudinal study. Arch Gen Psychiatry 2009;66:181-8.

59 Temmel CS, Rhodes R. Correlates of sedentary behaviour in children and adolescents aged 7-18: a systematic review. Health Fit J Can 2013;6:119-99.

60 Tremblay MS, Leblanc AG, Janssen I, et al. Canadian sedentary behaviour guidelines for children and youth. Appl Physiol Nutr Metab 2011;36:59-64.

61 Welk GJ, Corbin CB, Dale D. Measurement issues in the assessment of physical activity in children. Res Q Exerc Sport 2000;71(2 Suppl):S59-73.

62 Marshall SJ, Biddle SJ, Gorely T, et al. Relationships between media use, body fatness and physical activity in children and youth: a meta-analysis. Int I Obes Relat Metab Disord 2004;28:1238-46.

63 He FJ, Nowson CA, MacGregor GA. Fruit and vegetable consumption and stroke: meta-analysis of cohort studies. Lancet 2006;367:320-6.

64 Brown HE, Pearson N, Braithwaite RE, et al. Physical activity interventions and depression in children and adolescents: a systematic review and meta-analysis. Sports Med 2013:43:195-206

65 Liu M, Wu L, Ming Q. How does physical activity intervention improve self-esteem and self-concept in children and adolescents? Evidence from a meta-analysis. PLoS ONE 2015;10:e0134804 